nicht mehr auftreten, wenn die Halbwertsbreite der Teilchengrößenverteilung größer als $2 \%$ vom mittleren Radius wird. Bei dieser Halbwertsbreite ist eine Tröpfchengrößenstreuung von $\pm 3 \%$ schon nur noch mit der zu vernachlässigenden Häufigkeit von $N / 100$ vorhanden, wenn man mit $N$ die Häufigkeit des Auftretens des mittleren Radius bezeichnet.

Sollen also Aerosole die HOTS aufweisen, so darf die gesamte Streuung der Teilchengrößen höchstens $\pm 3 \%$ des mitleren Radius betragen und nicht wie bisher verschiedentlich angenommen wurde \pm 10 Prozent 12, 13.

12 V. K. LaMer, Air Pollution, McGraw-Hill-Book-Comp., New York, London 1952, Kap. 74.
Das scheint eine gewisse Antwort auf die von Koppe ${ }^{14} 1959$ veröffentlichte Theorie der Korngrößenverteilungen zu sein, wonach aus der Schwankung der Keimzahldichte beim Vorgang der Kondensation von Flüssigkeitsaerosolen aus der Dampfphase zwar eine relative Streuung von $1 \%$ zu erklären ist, jedoch nicht eine solche von 10 Prozent.

Meinem hochverehrten Lehrer, Herrn Professor Dr. H. Engelhard danke ich herzlich für die Anregung zu dieser Arbeit und sein großes Interesse an ihrem Fortschreiten.

13 L. LASSEx, unveröff. Dissertation, Heidelberg 1958.

14 H. Koppe, Z. Physik 156, 122 [1959].

\title{
Über das Grenzflädhenverhalten essentieller Fettsäuren und einiger ihrer Stereoisomeren
}

\author{
Von W. Wachs, H. Schwarz * und K. Zürcher ** \\ Aus dem Institut für Lebensmittelchemie und Lebensmitteltechnologie \\ der Technischen Universität Berlin-Charlottenburg (Leiter: Prof. Dr.-Ing. J. Schormüller) \\ (Z. Naturforschg. 15 b, 646-653 [1960]; eingegangen am 9. August 1960)
}

\begin{abstract}
In der vorliegenden Arbeit wird untersucht, ob die sich physiologisch unterschiedlich verhaltenden stereoisomeren Formen der essentiellen Linol- und Linolensäure auch ein unterschiedliches Grenzflächenverhalten zeigen. Zu diesem Zwecke wurden die $a$-Monoglyceride der all-cis-, cis-trans-, und all-trans-Linolsäure sowie der all-cis- und all-trans-Linolensäure dargestellt und auf ihre grenzflächenspannungserniedrigende Wirkung, ihr Assoziations-Verhalten sowie die Strukturviskosität und Stabilität mit ihnen hergestellter Emulsionen geprüft. Es ergab sich, daß in jedem Fall die trans-Verbindungen stärkere Grenzflächeneigenschaften besitzen als die korrespondierenden cis-Formen.
\end{abstract}

Die in der Natur vorkommenden Öle und Fette enthalten eine Anzahl vorwiegend ungesättigter Fettsäuren, die eine besondere biologische Bedeutung besitzen. Dazu gehören die essentiellen Fettsäuren, die als Zellbausteine wichtige physiologische Funktionen ausüben. Sie unterscheiden sich von den übrigen Fettsäuren nur durch die Anzahl und Stellung ihrer Doppelbindungen, können aber vom menschlichen Organismus nicht synthetisiert werden ${ }^{* * *}$. Als essentielle Säuren wurden bisher Linol-, Linolen- und Arachidonsäure erkannt. Fehlen diese Säuren in der tierischen Ernährung, so können Mangelsymptome auftreten, wie krankhafte Veränderungen der Haut und Nieren, erhöhte Wasserverdampfung durch die Haut, Wachstumsstillstand, Verringerung der Fortpflanzungsfähigkeit und erhöhte Mortalität. Beim Menschen wurden derartige ausgeprägte Man-

* H. Schwarz, Diss., Techn. Univ. Berlin 1959.

** K. Zürcher, Diss., Techn. Univ. Berlin 1959.

** Nach Thомasson ${ }^{1}$ ist das Vorhandensein von Doppelbindungen in der 6.7- sowie in der 9.10-Stellung von der endständigen Methylgruppe aus gerechnet für die essentiellen Eigenschaften dieser Verbindungen von ausschlag. gebender Bedeutung. gelerscheinungen bisher nicht beobachtet, jedoch scheint ein enger Zusammenhang zwischen der Zufuhr an essentiellen Fettsäuren und dem Cholesterinstoffwechsel zu bestehen. So konnte durch tägliche Verabreichung von Ölen mit einem hohen Gehalt an essentiellen Fettsäuren eine bedeutende Erniedrigung des SerumcholesterinSpiegels erzielt werden ${ }^{2}$. Bei Abwesenheit von essentiellen Fettsäuren entstehen in verstärktem Maße Cholesterinester gesättigter Fettsäuren, die zur Abscheidung neigen. Außerdem werden essentielle Fettsäuren zur Synthese von Phospholipiden benötigt, die ihrerseits für den Transport von Cholesterinester unentbehrlich sind ${ }^{3}$. Diese Verhältnisse werden vor allem in Verbindung mit der Arteriosklerose diskutiert ${ }^{4,5}$.

Da die essentiellen Fettsäuren auf Grund ihrer Doppelbindungen bei der Verarbeitung der Öle und Fette

1 H. J. Thomasson, Int. Rev. Vitamin Res. 25, 62 [1953].

2 K. Lang, in: Die ernährungsphysiologischen Eigenschaften der Fette, Seite 111. Steinkopff-Verlag, Darmstadt 1958.

3 R. B. Alfin-Slater, J. Amer. Oil Chemists' Soc. 34, 574 [1957].

4 J. B. Brown, Soybean Digest, Nov. 1957, 6.

5 R. B. Alfin-Slater, L. Aftergood, A. F. Wells u. H. J. Deuel, Arch. Biochem. Biophysics 52, 180 [1954]. 
in verschiedene isomere Formen übergehen können, sind sie für die moderne Fettechnologie von besonderem Interesse. Wie ir-spektrophotometrische Untersuchungen der letzten Jahre zeigten, kommen sie in der Natur fast ausschließlich in der cis-Form vor, während nach gewissen chemischen Eingriffen größere Mengen von transund Stellungsisomeren festzustellen sind. Diese Tatsache ist sehr wesentlich, da z. B. in bezug auf die Biotinwirksamkeit und die bakteriostatische Wirkung stellungsisomerer Octadecensäuren eine deutliche Überlegenheit der cis-Form gegenüber der trans-Form besteht ${ }^{6}$.

Ebenso zeigen nach Privetr ${ }^{7}$ die stereoisomeren Formen der Linol- und Linolensäure eine unterschiedliche Wirksamkeit. So besitzt bereits die cis-9-trans-12-Linolsäure keine biologische Aktivität mehr. Nach Holman ${ }^{8}$ ist die all-trans-Linolsäure vollkommen unwirksam gegenüber Mangelerscheinungen bei der Ratte, die konjugierte Form soll sogar toxisch wirken. Die Inaktivität im biologischen Sinne kommt ebenfalls bei den cis-transund all-trans-Formen darin zum Ausdruck, daß sie nach LANG $^{2}$ im Gegensatz zur all-cis-Verbindung nicht von der Lipoxydase angegriffen werden. Trans-Linolsäure hemmt sogar die Oxydation der cis-Linolsäure durch die Lipoxydase kompetitiv.

Aus ernährungsphysiologischen Gründen muß daher den Isomerie-Erscheinungen der ein- und mehrfach ungesättigten $\mathrm{C}_{18}$-Säuren große Beachtung geschenkt werden. Durch die cis- und trans-Konfiguration bedingt zeigen sie unterschiedliche physikalische Eigenschaften. So liegen z. B. die Schmelzpunkte der trans-Octadecensäuren ca. $15-30{ }^{\circ} \mathrm{C}$ höher als die der korrespondierenden cis-Formen. Die gleiche Erscheinung ist auch bei den stereoisomeren Formen der Linol- und Linolensäure zu beobachten. Die Schmelzpunkteigenschaften der Säuren übertragen sich auch auf ihre Triglyceride bzw. deren partielle Abbauprodukte, die Di- und Monoglyceride. Daß der Schmelzpunkt eines Fettes einen großen Einfluß auf die Verdaulichkeit und damit auf den Fettmetabolismus ausübt, ist bekannt.

Dieser Zusammenhang zwischen Konstitution und physiologischer Wirkung legt die Vermutung nahe, daß dabei Unterschiede im physikalisch-chemischen Verhalten eine entscheidende Rolle spielen. $\mathrm{Da}$ es sich beim Fettstoffwechsel um heterogene Reaktionen handelt, muß den Grenzflächenerscheinungen eine ganz besondere $\mathrm{Be}$ deutung beigemessen werden, denn sowohl die Resorption der enzymatisch abgebauten Triglyceride als auch der Transport der Lipoide im Organismus setzen bestimmte Grenzflächenverhältnisse voraus, die in erster Linie durch Assoziation der hydrophoben Fettsäureester

6 D. Melnik, H. J. Deuel, J. Amer. Oil Chemists' Soc. 31, 63 [1954].

7 O. S. Privett, F. J. Pusch u. R. T. Holman, Arch. Biochem. Biophysics 57, 156 [1955].

8 R. T. Holman u. E. Aaes-Jorgensen, Proc. Soc. exp. Biol. Med. 93, 175 [1956].

9 D. G. Dervichian, Olii minerali, grassi e saponi, colori e vernici 35, 229 [1958] ; C. A. 52, 21167 [1958].

10 L. de Bernard u. D. G. Dervichian, Bull. Soc. Chim. biol. 37, 943 [1955]. mit hydrophilen Verbindungen erreicht werden. So werden die Lipide im Verdauungstraktus nicht nur durch Assoziation mit Eiweißkörpern, sondern auch durch intermolekulare Assoziation in Lösung gehalten, wobei sich Mizellen verschiedener Art bilden. Dervichian ${ }^{9}$ konnte Assoziate von Fettsäuren, Phosphatiden und Cholesterin in stöchiometrischen Verhältnissen nachweisen. Auch de Bernard 10,11 stellte bei Lipiden (Triglycerid und Lecithin-Cholesterin) verschiedene Molekülassoziate fest. Man muß daher neben den bekannten Grenzflächenerscheinungen der Fettabbauprodukte auch der Neigung der Lipide zur Assoziation besondere Aufmerksamkeit widmen.

Die vorliegende Arbeit beschäftigt sich mit dem Grenzflächenverhalten essentieller Fettsäuren und einiger ihrer Stereoisomeren. Für diese Untersuchungen wurden ihre $a$-Monoglyceride herangezogen, da sie auf Grund ihres günstigen hydrophilen-lipophilen Gleichgewichts und damit ihrer guten Grenzflächeneigenschaften besonders geeignet erscheinen. Es sollte der Einfluß der Zahl der Doppelbindungen sowie der Stereoisomerie von Linol- und Linolensäure auf ihr Grenzflächenverhalten untersucht werden.

\section{Präparativer Teil}

Es waren zunächst die reine all-cis-Linolsäure und all-cis-Linolensäure herzustellen, die als Ausgangsmaterial für die entsprechenden all-trans-Verbindungen bzw. für die Darstellung der $a$-Monoglyceride dienten. Die cis-trans-Linolsäure wurde gesondert dargestellt.

\section{A. Darstellung der all-cis-Säuren}

Als Ausgangsmaterial diente für die Linolsäure Mohnöl, für die Linolensäure Leinöl. Es wurden zunächst die Methylester dieser Säuren hergestellt ${ }^{12}$, diese durch Ausfällung der gesättigten Fettsäureester als Harnstoff-Einschlußverbindungen angereichert und anschließend das Konzentrat durch fraktionierte Destillation im Hochvakuum angereichert.

$$
\text { B. Darstellung der all-trans-Säure n }
$$

Zur Umlagerung der all-cis-Säuren in die entsprechenden all-trans-Isomeren wurde nach KAss und BurR ${ }^{13,14}$ mit Selen gearbeitet und die umgelagerten Ester durch Destillation im Hochvakuum angereichert.

\section{Darstellung der cis-trans-Linolsäu re}

Als Ausgangsmaterial diente Ricinusöl, das nach Desnuelle und Masoni ${ }^{15}$ mit Kaliumbisulfat bei $240^{\circ} \mathrm{C}$

11 L. de Bernard, Bull. Soc. Chim. biol. 40, 161 [1958].

12 W. W. Westerfiled, Biochemical Preparations, Vol. 4, S. 86. Chapman \& Hall Ltd., New York-London 1955.

13 J. P. Kass u. G. O. Burr, J. Amer. chem. Soc. 61, 1062 [1939].

14 J. P. Kass, G. O. Burr u. J. Nichols, J. Amer. chem. Soc. 63, 1060 [1941].

15 P. Desnuelle u. R. Massoni, Bull. Soc. Chim. France, Documentat. 5, 1180 [1950]. 
dehydratisiert und anschließend in die Methylester übergeführt wurde. Dabei bildet sich ein Isomerengemisch, das vorzugsweise Isolensäureester enthält, die sich durch fraktionierte Destillation im Hochvakuum weiter anreichern lassen ${ }^{\mathbf{1 6}}$, da sie etwas niedriger sieden, als die ebenfalls entstehenden konjugierten Ester. Sie enthalten cis-cis- und cis-trans-Isomere, aus denen sich durch fraktionierte Tieftemperatur-Kristallisation die cis-9-trans-12-Linolsäure ziemlich rein gewinnen läßt ${ }^{17}$.

D. Darstellung der $\alpha$-Monoglyceride

Zur Darstellung der a-Monoglyceride der all-cis-, cis-trans-, all-trans-Linolsäure sowie der all-cis- und all-trans-Linolensäure aus den gereinigten Methylesterkonzentraten mußten zunächst die Fettsäuren freigesetzt, chloriert, destilliert und mit Acetonglycerin umgesetzt werden. Es wurde nach folgendem Schema gearbeitet:<smiles>[R]O[Cl+][Cl+][O-]</smiles><smiles>[R]OCOC1COC(C)(C)O1</smiles>

Dieses Verfahren ist besonders schonend und gibt nahezu quantitative Ausbeuten.

Zur Darstellung der Säurechloride aus den sehr empfindlichen mehrfach ungesättigten Fettsäuren eignet sich besonders Oxalchlorid ${ }^{18-20}$, wobei nach Wood ${ }^{21}$ nur in ganz geringem Umfang Umlagerungen eintreten. Das Acetonglycerin wurde nach VERKADE ${ }^{22}$ dargestellt und die anschließende Umsetzung mit den Säureglyceriden nach Daubert und Baldwin ${ }^{23}$ durchgeführt, wobei im Endgang die Wiederabspaltung des Acetons erfolgt. Die so erhaltenen $a$-Monoglyceridpräparate wurden durch Tieftemperatur-Kristallisation aus Aceton weiter gereinigt ${ }^{24,25}$. Es konnten auf diesem Wege nahezu 100-proz. Monoglyceride erhalten, jedoch geringe Reste anderer Stereoisomerer nicht völlig entfernt werden. Für unsere vergleichenden Untersuchungen erwies sich der Reinheitsgrad als ausreichend.

$$
\begin{aligned}
& \text { E. Reinheitsprüfung } \\
& \text { der } a-M \text { onoglyceride }
\end{aligned}
$$

\section{Bestimmung der Jodzahl nach der Halbmikromethode von Kaufmann 26}

$\begin{array}{lcc}\text { a-Monoglycerid der } & \text { Theoret. Jodzahl } & \text { Gef. Jodzahl } \\ \text { all-cis-Linolsäure } & 143,3 & 140,9 \\ \text { cis-trans-Linolsäure } & 143,3 & 135,9 \\ \text { all-trans-Linolsäure } & 143,3 & 136,5 \\ \text { all-cis-Linolensäure } & 216,2 & 208,2 \\ \text { all-trans-Linolensäure } & 216,2 & 194,4\end{array}$

17 J. E. Jackson, R. F. Paschke, W. Tolberg, H. M. Boyd u. D. H. Wheeler, J. Amer. Oil Chemists' Soc. 29, 229 [1952].

16 D. E. Terry u. D. H. WheELer, Oil and Soap 23, 88 [1946].

18 S. T. Bauer, J. Amer. Oil Chemists' Soc. 23, I [1946].

19 H. P. Kaufmann, Fette u. Seifen 59, 946 [1957].

20 H. P. Kaufmann, Fette u. Seifen 60, 85 [1958].

21 R. T. Wood, F. L. Jackson, A. R. Baldwin u. H. E. LongenECKER, J. Amer. chem. Soc. 66, 287 [1944].

22 P. E. Verkade u. J. Van der Lee, Receuil Trav. chim. Pays Bas 55, 271 [1936].

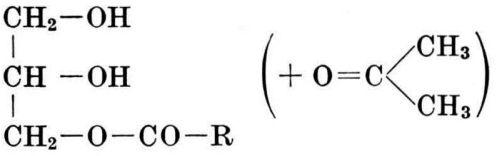

Bestimmung des a-Monoglyceridgehaltes nach PoHLE und Menlenbacher ${ }^{27}$ mit Perjodsäure

a-Monoglycerid der

Monoglyceridgehalt

all-cis-Linolsäure

[\%]

cis-trans-Linolsäure

99,8

all-trans-Linolsäure

100,7

all-cis-Linolensäure

99,8

99,2

all-trans-Linolensäure

100,2

Eine Aufnahme der IR-Spektren zeigte die für die trans-Verbindungen charakteristischen Absorptionsmaxima bei den spezifischen Wellenzahlen.

Die Schmelzpunkte der $a$-Monoglyceride wurden zur Reinheitsbestimmung nicht herangezogen, da sie wegen des Auftretens polymorpher Formen und der geringen thermischen Leitfähigkeit keine einheitlichen Werte ergeben $^{28}$.

\section{Meßtechnischer Teil}

$$
\text { 1. Messung der }
$$

Grenzflächenspannungs-Erniedrigung

Die große Grenzflächenaktivität der Monoglyceride, die durch ihren polaren Bau gegeben ist, bedingt eine starke Anreicherung und eine Ausrichtung der Moleküle in der Grenzfläche. Daher müssen Konstitutions-

23 B. F. Daubert u. A. R. Baldwin, J. Amer. Oil Chemists' Soc. 66, 997 [1944].

24 D. K. Kolb u. J. B. Brown, J. Amer. Oil Chemists' Soc. 32, 357 [1955].

25 J. B. Brown, J. Amer. Oil Chemists' Soc. 32, 646 [1955].

26 H. P. Kaufmann u. L. Hartweg, Ber. dtsch. chem. Ges. 70, 2554 [1937].

27 W. D. Pohle u. V. C. Mehlenbacher, J. Amer. Oil Chemists' Soc. 27, 54 [1950].

28 A. E. Bailey, Melting and Solidification of Fats. Interscience Publ., Inc., New York 1950. 
Isomerien in der Größe der GrenzflächenspannungsErniedrigung eines Öl/Wasser-Systems zum Ausdruck kommen. Von den in Betracht kommenden Methoden zur Messung der Grenzflächenspannung wurde die Ringabreißmethode gewählt, die bereits von amerikanischen Autoren und von uns zu ähnlichen Messungen verwendet wurde 29, 30. Als Meßinstrument diente eine Interfacialtensiometer nach Le c om te du Nouy (Fa. Krüss, Hamburg). Die Messung der GrenzflächenspannungsErniedrigung der untersuchten Substanzen erfolgte im System Baumwollsaatöl/Wasser bei $70^{\circ} \mathrm{C}$ in Abhängigkeit von steigenden Zusätzen an $\alpha$-Monoglycerid. Die Fehlergrenze der Messungen lag im Durchschnitt bei $\pm 0,5 \mathrm{dyn} / \mathrm{cm}$. Als Beispiel sei Tab. 1 angeführt. Die Ergebnisse bringt Abb. 1 .

\begin{tabular}{|c|c|c|c|}
\hline $\begin{array}{c}\text { Substanz } \\
{[\mathrm{mg}]}\end{array}$ & $\begin{array}{c}\text { Baumwoll- } \\
\text { saatöl } \\
{[\mathrm{g}]}\end{array}$ & $\begin{array}{c}\text { Monoglycerid } \\
\text { im Baum- } \\
\text { wollsaatöl } \\
{[\%]}\end{array}$ & $\begin{array}{c}\text { Grenzflächen- } \\
\text { spannung } \\
\text { [dyn/cm] }\end{array}$ \\
\hline 20,8 & 10,0243 & 0,20 & 22,9 \\
62,5 & 10,0028 & 0,62 & 19,0 \\
97,3 & 10,1864 & 0,96 & 16,5 \\
152,5 & 10,1838 & 1,49 & 14,2 \\
199,5 & 10,3079 & 1,93 & 12,4 \\
256,4 & 10,1456 & 2,52 & 10,8 \\
307,1 & 10,1423 & 3,02 & 9,5 \\
377,2 & 10,2139 & 3,68 & 8,2 \\
397,2 & 10,0370 & 3,95 & 7,6 \\
512,5 & 10,0370 & 4,96 & 5,8 \\
\hline
\end{tabular}

Tab. 1. Grenzflächenspannungs-Erniedrigung von all-cis-Linolsäure- $\alpha$-monoglycerid.

Die erhaltenen Kurven lassen eine Abnahme der Grenzflächenspannung in der Reihenfolge all-cis-, cistrans-, all-trans-Linolsäure- $\alpha$-monoglycerid sowie all-ciszu all-trans-Linolensäure- $\alpha$-monoglycerid erkennen.

\section{B e stim mung \\ des Assoziationsgrades}

Da die mechanische Stabilität des Grenzflächenfilms in erster Linie durch Assoziation bedingt ist, müssen bei den stereoisomeren Monoglyceriden auch Unterschiede im Assoziationsgrad auftreten. Da zwischen der Assoziation der Moleküle in der Grenzfläche und im Innern der Lösung eine enge Beziehung besteht ${ }^{10}$, verwendeten wir zur Bestimmung des Assoziationsgrades der untersuchten a-Monoglyceride die Dialysenmethode nach J ANDER und Spandau ${ }^{31,32}$, (vgl. auch l. c. ${ }^{33}$ ). Dabei wurde in einem unpolaren, mit Wasser versetzten Lösungsmittel (Dioxan/Wasser) bei $20{ }^{\circ} \mathrm{C}$ gearbeitet und die Dialysegeschwindigkeit interferometrisch verfolgt, wie in einer früheren Veröffentlichung beschrieben. Die Meßergeb-

29 A. T. Gros u. R. O. Feuge, J. Amer. Oil. Chemists' Soc. 28, 1 [1951].

30 H. Schwarz, Diplom-Arbeit, Techn. Univ. Berlin-Charlottenburg 1956.

31 G. Jander u. H. Spandau, Z. physik. Chem. Abt. A 185, 326 [1939].

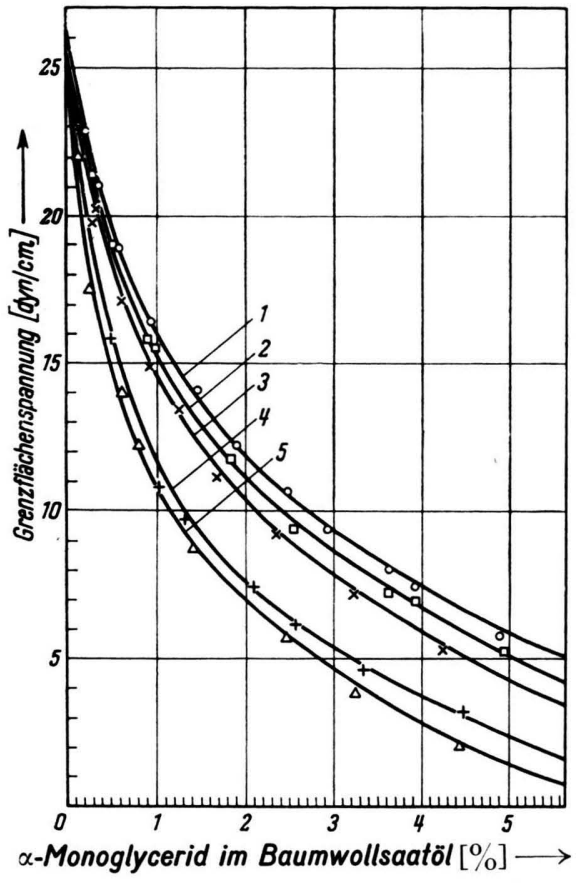

Abb. 1. Grenzflächenspannung in Abhängigkeit vom Monoglyceridgehalt.

nisse in Tab. 2 sind als Mittelwerte der Teilchenwerte anzusehen, da neben Assoziaten auch dissoziierte Moleküle vorliegen.

\begin{tabular}{|l|l|c|c|}
\hline \multicolumn{1}{|c|}{$\alpha$-Monoglycerid der } & $\begin{array}{c}\text { Mittl. } \\
\text { Teilchengew. }\end{array}$ & $\begin{array}{c}\text { rel. } \\
\text { Fehler } \\
\%\end{array}$ & $\alpha^{*}$ \\
\hline all-cis-Linolsäure & $1164 \pm 16$ & 1,4 & 3,3 \\
cis-9-trans-12-Linolsäure & $1251 \pm 40$ & 3,2 & 3,5 \\
all-trans-Linolsäure & $2757 \pm 100$ & 3,7 & 7,8 \\
all-cis-Linolensäure & $3721 \pm 30$ & 0,8 & 10,6 \\
all-trans-Linolensäure & $\mathbf{4 7 9 5} \pm \mathbf{5 0}$ & 1,1 & $\mathbf{1 3 , 6}$ \\
\hline
\end{tabular}

Tab. 2. Mittlere Teilchengewichte der $\boldsymbol{\alpha}$-Monoglycerid-Assoziate nach der Dialysenmethode. * Der Assoziationsgrad $\alpha$ ist der Quotient aus gefundenem und theoretischem Teilchengewicht.

Danach steigen die Teilchengewichte in der Reihenfolge all-cis-, cis-trans-, zu all-trans-Linolsäure- $\alpha$-monoglycerid und all-cis- zu all-trans-Linolensäure- $\alpha$-monoglycerid an. Es besitzt also jeweils die cis-Form das niedrigere Teilchengewicht. Außerdem zeigen die a-Monoglyceride der 3-fach ungesättigten Säuren ein

32 H. Spandau, Teilchengewichts-Bestimmung organischer Verbindungen mit Hilfe der Dialysenmethode. Monographie Nr. 63 zu "Angewandte Chemie“, Verl. Chemie, Weinheim 1951.

33 W. Wachs u. J. Wendenburg, Kolloid-Z. 156, 35 [1958]. 
fast doppelt so großes Teilchengewicht, als die der 2-fach ungesättigten Säuren (Tab. 3).

\begin{tabular}{|c|c|c|}
\hline $\begin{array}{c}\text { Lage der } \\
\text { Doppelbindung }\end{array}$ & $\begin{array}{c}\text { Teilchengewicht } \\
\text { cis-Form }\end{array}$ & $\begin{array}{c}\text { Teilchengewicht } \\
\text { trans-Form }\end{array}$ \\
\hline $9.10-12.13$ & 1164 & 2757 \\
$9.10-12.13-15.16$ & 3721 & 4795 \\
\hline
\end{tabular}

Tab. 3. Mittlere Teilchengewichte der $\alpha$-Monoglyceride zweiund dreifachungesättigter Fettsäuren.

\section{Bestimmung der Strukturviskosität}

Die im folgenden beschriebenen Untersuchungen wurden in der Erwartung durchgeführt, daß das verschiedenartige Grenzflächenverhalten der $\alpha$-Monoglyceride auch zur Bildung von Emulsionen unterschiedlicher Strukturviskosität führt. So gestatten z. B. Messungen der Oberflächenviskosität Einblicke in die Struktur des

\begin{tabular}{|c|c|}
\hline $\begin{array}{c}\text { Schergefälle } \\
{\left[\mathrm{sec}^{-1}\right]}\end{array}$ & $\eta(\mathrm{cp})$ \\
\hline 6,79 & 5417 \\
13,50 & 3070 \\
20,30 & 2306 \\
40,70 & 1365 \\
60,60 & 1011 \\
122,00 & 612 \\
184,00 & 462 \\
366,00 & 378 \\
550,00 & 234 \\
1100,00 & 154 \\
\hline
\end{tabular}

Tab. 4. Strukturviskosität einer all-cis-Linolsäurea-monoglycerid-Emulsion.

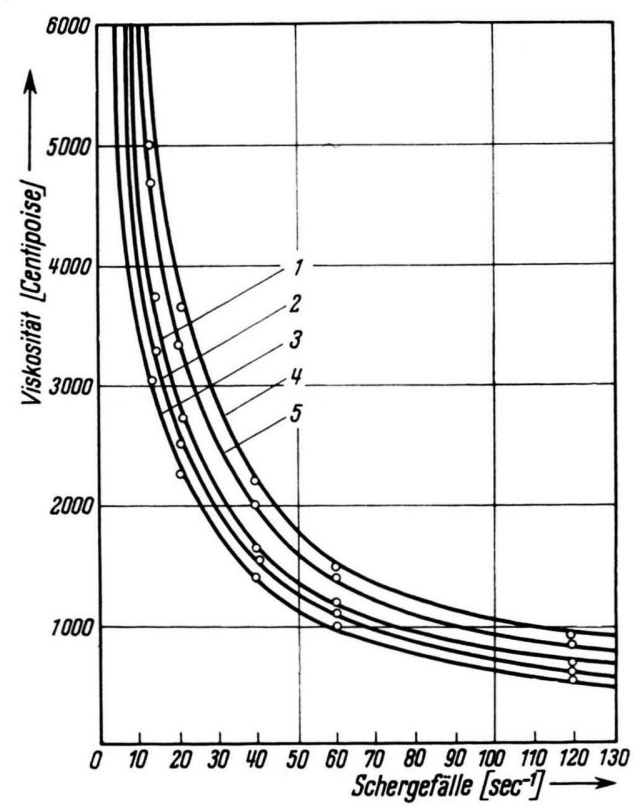

Abb. 2. Strukturviskosität in Abhängigkeit von der Konfiguration der zugesetzten Monoglyceride.
Oberflächenfilms ${ }^{34}$. Zu diesem Zweck wurden mit den a-Monoglyceriden Wasser-in-Petroleum-Emulsionen hergestellt und die Strukturviskosität dieser Emulsionen mit Hilfe eines Rotationsviskosimeters (Rotavisko, Fa. Haake, Berlin) gemessen. Die Herstellung der Emulsionen erfolgte mittels eines durch einen Synchronmotor $(3500 \mathrm{U} / \mathrm{min})$ angetriebenen Evolventenrührers $(\varnothing 30 \mathrm{~mm})$. Bei den Messungen wurden die normale Temperierung und der Drehkörper I $(\varnothing 40,0 \mathrm{~mm})$ benutzt. Aus 2-proz. a-Monoglyceridlösungen in Petroleum wurden mit auf $15{ }^{\circ} \mathrm{C}$ gekühltem Wasser Emulsionen von $18{ }^{\circ} \mathrm{C}$ hergestellt. Das Volumenverhältnis Petroleum : Wasser betrug 10:1 und die Messung erfolgte bei $20^{\circ} \mathrm{C}$. Als Beispiel sei Tab. 4 angeführt.

Die Strukturviskosität der untersuchten Emulsionen (Abb. 2) steigt demnach sowohl bei Linolsäure- als auch bei Linolensäure- $\alpha$-monoglycerid von der all-cis-Form zur trans-Form an, entsprechend der Zunahme der Grenzflächenaktivität dieser Verbindungen.

\section{Bestimmung der Stabilität}

Die Unterschiede in der mechanischen Stabilität des Grenzflächenfilms, wie sie die Messungen der Strukturviskosität erkennen lassen, muß sich auch auf die Stabilität der Emulsionen auswirken.

Im vorliegenden Fall wird die Stabilität der Emulsionen nach dem Aufrahmungsverfahren von YeadoN ${ }^{35}$ zeitlich verfolgt. Die Steigung der Kurven gibt die Anzahl $\mathrm{ml}$ organischer Phase an, die in der Zeiteinheit abgeschieden werden. Der reziproke Wert $K$ ist als $\mathrm{Maß}$ für die Emulsionsstabilität anzusehen, da er die Anzahl der Minuten bezeichnet, die zur Abtrennung eines ml notwendig sind. Je größer $K$ ist, desto stabiler muß die Emulsion sein. Nach Ermittlung der günstigsten Versuchsbedingungen wurden jeweils 1-proz. Lösungen der a-Monoglyceride in Petroleum hergestellt und $20 \mathrm{ml}$ der Petroleumlösungen wie bei den StrukturviskositätsMessungen unter Zutropfen von $25 \mathrm{ml}$ dest. Wasser $\left(20^{\circ} \mathrm{C}\right)$ emulgiert. Die erhaltenen Emulsionen wurden in enge graduierte Glaszylinder mit Glasstopfen gefüllt

\begin{tabular}{|c|c|}
\hline Zeit & $\begin{array}{c}\text { abgeschiedenes } \\
\text { Petroleum } \\
{[\mathrm{ml}]}\end{array}$ \\
\hline $\mathrm{min}]$ & 4,0 \\
10 & 6,5 \\
15 & 9,1 \\
20 & 11,0 \\
25 & 12,0 \\
30 & 13,1 \\
35 & 14,1 \\
40 & 15,0 \\
45 & 15,3 \\
50 & 16,2 \\
\hline
\end{tabular}

Tab. 5. Stabilität einer all-cis-Linolsäure- $a$-monoglyceridEmulsion.

34 G. E. Boyd u. F. VAskov, J. Colloid Sci. 13, 275 [1958].

35 D. A. Yeadon, L. A. Goldblatt u. A. M. Altschul, J. Amer. Oil Chemists' Soc. 8, 435 [1958]. 


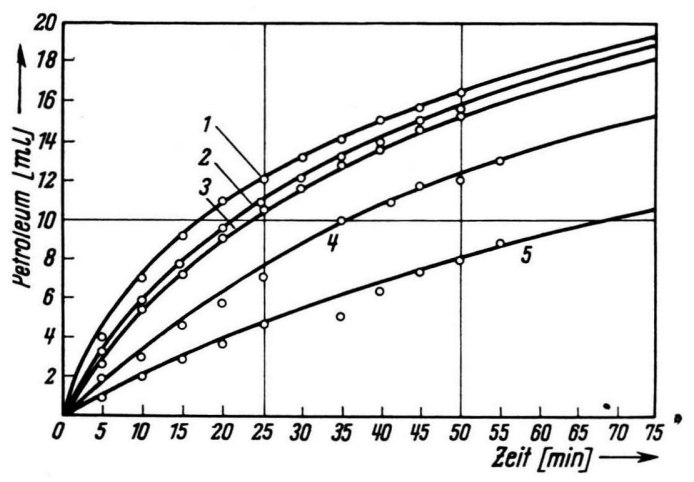

Abb. 3. Emulsionsstabilität in Abhängigkeit von der Konfiguration der zugesetzten Monoglyceride.

und die Entmischung bei $20^{\circ} \mathrm{C}$ zeitlich verfolgt. Als Beispiel sei Tab. 5 angeführt.

Wie aus den Meßwerten (Abb. 3) zu ersehen ist, entmischen sich die Emulsionen mit a-Monoglyceriden, welche trans-Doppelbindungen enthalten, etwas langsamer als die der entsprechenden cis-Verbindungen.

\section{Diskussion}

Grenzflächenspannungs - Erniedri gung. Die Ergebnisse (Abb. 1) lassen erkennen, daß die trans-Verbindungen eine größere Grenzflächenaktivität besitzen als die korrespondierenden cis-Formen, und daß sowohl bei den cis- als auch bei den trans-Verbindungen mit wachsender Zahl der Doppelbindungen eine Zunahme der Grenzflächenaktivität festzustellen ist. Die untersuchten $\alpha$-Monoglyceride zeigen also eine steigende Grenzflächenaktivität in der Reihenfolge: all-cis-Linolsäure-, cis9-trans-12-Linolsäure-, all-trans-Linolsäure-, all-cisLinolensäure- und all-trans-Linolensäure- $\alpha$-monoglycerid.

Betrachten wir die Anreicherung der Monoglyceride in der Grenzfläche, so ergibt sich etwa folgendes Bild: die infolge der Wärmebewegung rasch an die Lipoid/Wasser-Grenzfläche diffundierenden Monoglyceridmoleküle werden dort mehr oder weniger fest adsorbiert unter Ausbildung eines Adsorptions-Desorptions-Gleichgewichtes. Die Lage des Gleichgewichtes und damit die Grenzflächenbesetzung werden vom Emulgatorenangebot und dem konstitutionsabhängigen hydrophilen-lipophilen Charakter des Emulgatormoleküls bestimmt. Es muß also bei den Messungen der stereoisomeren $\mathrm{C}_{18}$-Monoglyceride unter gleichen Konzentrations-Verhältnissen eine unterschiedliche Besetzung der Grenzfläche mit Emulgatormolekülen erfolgen. Da die Ausbil- dung eines monomolekularen Films zwischen lipoider und wäßriger Phase allgemein eine Senkung der Grenzflächenspannung auf Werte gegen Null zur Folge hat, kann aus dem Verlauf der $\sigma / c$-Kurven auf die Ausbildung des Grenzflächenfilms in Abhängigkeit von der Konstitution geschlossen werden.

Die Tatsache, daß die Monoglyceride der all-transSäuren die Grenzflächenspannung stärker erniedrigen als die entsprechenden all-cis-Verbindungen, wird durch Betrachtung des stereochemischen Baues verständlich (Abb. 4).
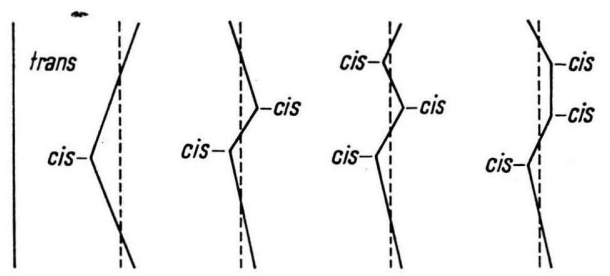

Abb. 4. Vereinfachte geometrische Formen von Öl-, Linolund Linolensäure.

Bei den Monoglyceriden der cis-Säuren, deren geknickte Kohlenwasserstoffketten eine enge Parallellagerung erschweren, können die Lipoidmoleküle besser zwischen den Paraffinketten eindringen (Einschluß-Solvatation) und man muß daher hier bevorzugt mit Solvatations-Erscheinungen rechnen, die eine große Erniedrigung der Grenzflächenspannung wie bei den trans-Verbindungen verhindern. Die Asymmetrie der Monoglycerid-Moleküle nimmt mit Einführung neuer Doppelbindungen nicht zu, sondern mehrere cis-Winkel gleichen sich gegenseitig aus, so daß die abgewinkelten Paraffinketten sich im Raum um eine gedachte Hauptachse winden. Dies steht in Übereinstimmung mit den Meßergebnissen, denn sowohl in der cis- als auch in der trans-Reihe steigt die Grenzflächenaktivität mit wachsender Zahl der Doppelbindungen. Darüber hinaus können auch die Doppelbindungen über elektronische Kräfte die hydrophilen Eigenschaften des Moleküls verstärken und somit seine Grenzflächenaktivität steigern.

Der unterschiedliche Bau der untersuchten Verbindungen muß neben ihrem Grenzflächenverhalten auch ihr Assoziationsvermögen beeinflussen.

Assoziations - Verhalten. Die Ergebnisse der Teilchengewichts-Bestimmungen nach der Dialysenmethode zeigen, daß diese in der Reihenfolge all-cis-Linolsäure-, cis-trans-Linolsäure-, all-transLinolsäure- sowie all-cis-Linolensäure-, all-transLinolensäure- $\alpha$-monoglycerid ansteigen. Außerdem 
erkennt man, daß jeweils die $c i s$-Form das niedrigere Teilchengewicht und demnach das geringere Assoziationsvermögen besitzt (Tab. 2,3).

Betrachten wir die Moleküle der all-trans- $\mathrm{C}_{18}$-Fettsäuren, so sehen wir, daß zwischen ihnen Assoziations-Erscheinungen auf zwei Arten möglich sein können:

1. Die Assoziation kann durch Dispersionskräfte unter Parallellagerung der Kohlenwasserstoffketten erfolgen. Hierfür ist eine entsprechende Annäherung der Moleküle erforderlich, da die Dispersionskräfte der 6. Potenz des Abstandes der Moleküle umgekehrt proportional sind. Eine derartige Annäherung ist natürlich bei den Säuren mit gestreckter Kette am größten.

2. Eine Assoziation der Monoglyceride ist aber auch zwischen den polaren Gruppen der Moleküle möglich, wenn es zur Ausbildung einer Wasserstoffbrückenbindung kommt*. Da die Monoester des Glycerins höhere Schmelzpunkte und größere Viskosität zeigen als die Ester derselben Säure mit einem einwertigen Alkohol, dürfte auch hier der Assoziation am polaren Teil der Moleküle eine gewisse Wahrscheinlichkeit zukommen **. Da aber bei den vorliegenden Untersuchungen der Glyceridrest der Verbindungen konstant gehalten wurde, können die Unterschiede im Assoziations-Verhalten nur durch den Fettsäurerest verursacht werden.

In Ubbereinstimmung mit den Meßergebnissen der Grenzflächenspannungen zeigt sich auch beim Assoziations-Verhalten der untersuchten Monoglyceride, daß bei gestrecktem Bau der Moleküle das Assoziations-Vermögen am größten ist, so daß die transFormen jeweils stärker assoziieren, als die cis-Verbindungen. Bei Zunahme der Doppelbindungen erhöht sich auch bei den cis-Verbindungen das Assoziations-Vermögen (gestreckterer Molekülbau).

Strukturvisk osität. Das verschiedenartige Assoziations-Verhalten der $\alpha$-Monoglyceride führt weiterhin zur Bildung von Emulsionen unterschiedlicher Strukturviskosität. Wie die an W/O-Emulsionen durchgeführten Messungen zeigen, steigt sowohl bei Linolsäure- als auch bei Linolensäure- $\alpha$-mono-

\footnotetext{
* So konnten Debeye u. Prins (J. Coll. Sci. 13, 86 [1958]) bei Untersuchungen über die micellare Dispersion von $\alpha$-Monoglyceriden der gesättigten Fettsäuren von $C_{10}-C_{18}$ durch IR-Spektren den Beweis für die Existenz intermolekularer Wasserstoffbindungen in benzolischen Micellen erbringen.
}

glycerid die Strukturviskosität von der all-cis-Form zur all-trans-Form entsprechend der Zunahme des Assoziationsgrades dieser Verbindungen an. Außerdem zeigen die Monoglyceride der Linolensäuren eine beträchtlich höhere Strukturviskosität als die der Linolsäuren. Diese Werte stehen ebenfalls in Übereinstimmung mit den Vorstellungen über Pakkung und Bau der Fettsäurefilme, die durch die Struktur der Kohlenstoffkette bedingt sind. Während die trans-Säuren feste Filme bilden, verhindert die cisDoppelbindung eine dichte Packung und ihr Film bleibt flüssig unter Drucken, bei welchen trans- und gesättigte Säuren fest sind.

Stabilität. Die mechanische Festigkeit des Grenzflächen-Films findet selbstverständlich auch ihren Ausdruck in der Stabilität der mit den zu untersuchenden Monoglyceriden hergestellten Emulsionen. Nach den Entmischungsdiagrammen (Abb. 3) ist die Stabilität der Emulsionen bei transIsomeren größer als bei den zugehörigen cis-Formen. Im übrigen steht die Stabilität der Emulsionen in direkter Relation zur Grenzflächenaktivität der verwendeten $\alpha$-Monoglyceride.

Die Ergebnisse unserer Untersuchungen zeigen demnach übereinstimmende Unterschiede zwischen den untersuchten stereoisomeren $\alpha$-Monoglyceriden von Linol- und Linolensäure, die auf ihrer unterschiedlichen Konfiguration beruhen.

Abschließend sei noch im Hinblick auf das anfangs angeführte Problem des Cholesterintransports im Organismus auf einige Ähnlichkeiten im Verhalten von trans- und gesättigten Fettsäuren hingewiesen. So besitzen die trans-Säuren im Verhältnis zu ihren cis-Isomeren einen wesentlich höheren Schmelzpunkt wie z. B. cis-Octadecensäure, Schmp. $16^{\circ} \mathrm{C}$, trans-Octadecensäure, Schmp. $43-44^{\circ} \mathrm{C}$, Stearinsäure, Schmp. $69,6^{\circ} \mathrm{C}$. Auch in den Grenzflächeneigenschaften zeigen die trans-Säuren stärkere Ähnlichkeit mit den gesättigten Fettsäuren. Während die $\sigma / c-K u r v e n$ von Stearinsäure- und trans-Linolensäure- $\alpha$-monoglycerid dicht beieinander liegen, zeigt die Kurve des $\alpha$-Ölsäuremonoglycerids eine wesentlich geringere Erniedrigung der Grenzflächenspannung. Auch die Assoziationsgewichte von trans-

\footnotetext{
** Auch eine Solvatation am unpolaren Teil der Monoglyceridmoleküle könnte durch Parallellagerung ihrer Kohlenwasserstoffketten und den Molekülen des lipiden Lösungsmittels erfolgen. Doch dürfte bei den trans-Isomeren nur eine geringe Solvatisierbarkeit vorliegen.
} 
Linolensäure- und Stearinsäure- $\alpha$-monoglycerid liegen mit 4700 und 4000 eng beieinander. So besteht eventuell die Möglichkeit, daß bei einem hohen Angebot an trans-Fettsäuren ebenfalls schwerer lösliche Cholesterinester auftreten.
Wir möchten an dieser Stelle dem Fonds der Chemischen Industrie für die Unterstützung unserer Arbeiten danken. Herrn Priv.-Doz. Dr.-Ing. G. KRESze vom Organisch-chemischen Institut der Techn. Universität danken wir für die Aufnahme der IRSpektren.

\title{
Ein Hemmstoff der Photosynthese-Phosphorylierung in Chloroplasten
}

\author{
Von Ulrich Heber \\ Aus dem Institut für Landwirtschaftliche Botanik der Universität Bonn \\ (Direktor: Prof. Dr. H. UllRICH) \\ (Z. Naturforschg. 15 b, 653-656 [1960]; eingegangen am 21. Juli 1960)
}

\begin{abstract}
In Chloroplasten von Vicia faba ssp. minor wurde ein Stoff gefunden, der die zyklische Photosynthese-Phosphorylierung von Spinat und Weizenchloroplasten stark zu hemmen vermag. ViciaChloroplasten selbst sind nicht in nennenswertem Umfange zur zyklischen Photosynthese-Phosphorylierung befähigt. Der Hemmstoff besitzt offenbar Lipoidnatur und ist an die Chloroplastenstruktur gebunden, kann aber durch Aceton extrahiert werden. Die mögliche Bedeutung des Hemmstoffes für Elektronentransport-Prozesse in den Chloroplasten wird hier im Hinblick auf festgestellte jahreszeitliche Schwankungen der Photosynthese-Phosphorylierung besprochen.
\end{abstract}

Seit mehreren Jahren ist die von Arnon und Mitarbb. ${ }^{1}$ bei Spinat-C* aufgefundene zyklische PP einer der Brennpunkte der Photosynthese-Forschung. In dieser Reaktion wird mit Hilfe der Lichtenergie freies anorganisches Phosphat mit ADP zu ATP verbunden, wobei kein Gaswechsel auftritt ${ }^{2}$. Das gebildete ATP zerfällt im Photosynthesezyklus unter Regenerierung von ADP bei der Reduktion der Phosphoglycerinsäure und der Phosphorylierung von Ribulosemonophosphat ${ }^{3}$. Somit gehört die PP zu den Grundprozessen der Photosynthese.

Jedoch gelang es Simonis und FüchtBauER ${ }^{4}$ nicht, die an Spinat-C erarbeiteten Befunde an Chloroplasten anderer Pflanzenspezies zu bestätigen. Diese zeigten keine zyklische PP. Jedoch fanden Simonis und FüchtBauer im löslichen Extrakt von $\mathrm{C}$ verschiedener Pflanzenarten, darunter auch Spinat, ein Prinzip, das die PP von Spinat-C um etwa 20\% hemmte. Es liegt also nahe, bei der mangelnden PP von $\mathrm{C}$ anderer Pflanzen als Spinat an Hemmstoffeinflüsse zu denken. Wir bearbeiten seit längerem das PhotosyntheseverhaIten von Vicia faba. Deren C zeigen nur sehr geringe oder keine zyklische PP. Dies ist offenbar auf die Existenz eines an die Chloroplasten gebundenen Hemmstoffes zurückzuführen,

1 D. I. Arnon, M. B. Allen u. F. R. Whatley, Nature [London] 174, 394. [1954].

- Als Abkürzungen werden im folgenden benutzt: $\mathrm{C}=\mathrm{Chlo-}$ roplasten, $\mathbf{P P}=$ Photosynthese-Phosphorylierung, $\mathbf{C R}=$ Chloroplastenrückstand, $\mathrm{CE}=$ Chloroplastenextrakt, TRIS $=$ Tris (hydromethyl) aminomethan, $\mathrm{ADP}=$ Adenosindiphosphat, ATP = Adenosintriphosphat. wie im folgenden dargelegt wird. Gleichzeitig geben die Ergebnisse Einblick in jahreszeitlich bedingte Schwankungen der Höhe der PP.

\section{Material und Methode}

Die zyklische PP wurde an $C$ von Spinat, Winterweizen (Sorten Hauter's II und Criewener 192) und Vicia faba ssp. minor gemessen, wobei die $\mathrm{C}$ der erstgenannten Pflanzenarten als Testobjekte für den in den Vicia-C festgestellten Hemmstoff dienten. Die Höhe der PP bestimmten wir unter den von JAGENDORF und Avron ${ }^{5}$ angegebenen Bedingungen mit Phenacinmethosulfat als Cofaktor. Im Gasraum der $0,5 \mathrm{~cm}$ breiten Reaktionsküvetten befand sich Luft. Der Einsatz von aktiven C aus Weizen oder Spinat betrug zwischen 100 und $150 \gamma$ Chlorophyll im 1,2-ml-Ansatz, der Zusatz von hemmstoffhaltigen Vicia-C oder von CR aus Vicia zwischen 200 und $300 \gamma$ Chlorophyll oder einer diesem äquivalenten Menge an extrahiertem $\mathrm{CR}_{\mathrm{H}}$. Als Beleuchtungsquelle dienten zwei Glühlampen zu je 200 Watt in $15 \mathrm{~cm}$ Entfernung zu beiden Seiten der gekühlten Küvette. Die Einzelergebnisse schwankten je nach verwendetem Material nicht unbeträchtlich, doch ist die Richtung der erhaltenen Ergebnisse deutlich erkennbar.

Die C wurden in einer Modifikation der von Avron und JAGENDORF (1958) angegebenen Methode isoliert. Als Isolationsmedium diente eine Lösung von 0,4-m. Saccharose, 0,01-m. NaCl, 0,005-m. Ascorbinsäure und

2 D. I. Arnon, Nature [London] 184, 10 [1959].

3 J. A. Bassham u. M. Calvin, The Path of Carbon in Photosynthesis, New Jersey 1957.

4 W. Simonis u. W. Füchtbauer, Planta 54, 95 [1959].

5 A. T. Jagendorf u. M. Avron, J. biol. Chemistry 231, 277 [1958]. 